\title{
Ladder Operators for Lamé Spheroconal Harmonic Polynomials ${ }^{\star}$
}

\author{
Ricardo MÉNDEZ-FRAGOSO †‡ and Eugenio LEY-KOO \\ † Facultad de Ciencias, Universidad Nacional Autónoma de México, México \\ E-mail: rich@ciencias.unam.mx \\ URL: http://sistemas.fciencias.unam.mx/rich/ \\ ¥ Instituto de Física, Universidad Nacional Autónoma de México, México \\ E-mail:eleykoo@fisica.unam.mx
}

Received July 31, 2012, in final form October 09, 2012; Published online October 17, 2012

http://dx.doi.org/10.3842/SIGMA.2012.074

\begin{abstract}
Three sets of ladder operators in spheroconal coordinates and their respective actions on Lamé spheroconal harmonic polynomials are presented in this article. The polynomials are common eigenfunctions of the square of the angular momentum operator and of the asymmetry distribution Hamiltonian for the rotations of asymmetric molecules, in the body-fixed frame with principal axes. The first set of operators for Lamé polynomials of a given species and a fixed value of the square of the angular momentum raise and lower and lower and raise in complementary ways the quantum numbers $n_{1}$ and $n_{2}$ counting the respective nodal elliptical cones. The second set of operators consisting of the cartesian components $\hat{L}_{x}, \hat{L}_{y}, \hat{L}_{z}$ of the angular momentum connect pairs of the four species of polynomials of a chosen kind and angular momentum. The third set of operators, the cartesian components $\hat{p}_{x}, \hat{p}_{y}, \hat{p}_{z}$ of the linear momentum, connect pairs of the polynomials differing in one unit in their angular momentum and in their parities. Relationships among spheroconal harmonics at the levels of the three sets of operators are illustrated.
\end{abstract}

Key words: Lamé polynomials; spheroconal harmonics; ladder operators

2010 Mathematics Subject Classification: 20C35; 22E70; 33C47; 33C80; 81R05

\section{Introduction}

One of our contributions to the Symposium on Superintegrability, Exact Solvability and Special Functions dealt with the topic of "Symmetries and asymmetries in quantum systems confined by elliptical cones", reviewing some of our results on the rotations of free asymmetric molecules $[9,10]$ and their extensions for other systems under confinement $[17,18,19]$. While the free systems involve Lamé spheroconal harmonic polynomial eigenfunctions, the systems confined by elliptical cones involve quasi-periodic Lamé eigenfunctions represented as infinite series, due to the breaking of the parity symmetry with respect the axis of the confining cone. Nevertheless, the methodology for the accurate and convergent evaluation of eigenvalues and eigenfunctions is the same. The differences reside in the boundary conditions, translating into recurrence relations of three terms and four terms for the expansion coefficients, respectively; such relations can be cast into matrix forms which are of finite size for the free systems [10] and of infinite size for the confined systems $[17,18,19]$ leading to the corresponding polynomial and infinite series representations. The latter can be evaluated with sufficient accuracy using finite and large enough size matrices, testing for convergence along the way.

Concerning the conditions of exact solvability requiring polynomial solutions and ladder operators connecting them, the Lamé spheroconal harmonics for the free systems satisfy the first

\footnotetext{
*This paper is a contribution to the Special Issue "Superintegrability, Exact Solvability, and Special Functions". The full collection is available at http://www.emis.de/journals/SIGMA/SESSF2012.html
} 
one and in this contribution three sets of ladder operators in the purely spheroconal formulation are reported. It must be pointed out that one of these sets had been identified sometime ago $[13,14,15,24,25]$ and our recent work [18] in its section "On Developing the Theory of Angular Momentum in Bases of Lamé Spheroconal Harmonics" identified a second set. However, it must also be admitted that in these treatments the connections with the spherical and cartesian harmonic bases were used.

The material in the manuscript is distributed in the following way: Section 2 reviews briefly the simultaneous separability of the square of the angular momentum operator and the asymmetry distribution Hamiltonian eigenvalue equations, the integration of the Lamé differential equation in the respective elliptical cone coordinates, illustrating the lower eigenvalues and eigenfunctions for their matched products making up the Lamé spheroconal harmonic polynomials. Section 3 identifies the raising and lowering actions on the numbers $n_{1}$ and $n_{2}$ counting the nodal elliptical cones in the neighboring spheroconal harmonic polynomials as their respective eigenvalues $h_{n_{1}}^{A}\left(k_{1}^{2}\right)$ and $h_{n_{2}}^{B}\left(k_{2}^{2}\right)$ are complementarily increased and decreased, which follows as a corollary to the tridiagonal matrix diagonalization method of solution, for chosen values of $\ell$ and species $A B$ of the polynomials. Section 4 identifies the shifting actions of the $\hat{L}_{x}, \hat{L}_{y}$ and $\hat{L}_{z}$ operators on the individual Lamé polynomials of species $A$ and $B$, respectively, and on their matched products of species $A B$ for each fixed value of $\ell$. Section 5 identifies the corresponding actions of the $\hat{p}_{x}, \hat{p}_{y}$ and $\hat{p}_{z}$ operators on an initial harmonic polynomial $\ell A B$, raising and lowering the angular momentum by one unit $\ell^{\prime}=\ell \pm 1$ into final polynomials $\ell^{\prime} A^{\prime} B^{\prime}$ of opposite parity and different species. Section 6 discusses the main results of this work, including relationships among the spheroconal harmonics in the three levels of the identified operators, as well as connections with other works in the literature.

\section{Eigenvalues and eigenfunctions of $\hat{L}^{2}$ and $\hat{H}^{*}$}

Kramers and Ittmann [6] pioneered the quantum mechanical study of the rotations of asymmetric molecules using spheroconal coordinates, showing the separability of the eigenvalue equations for the square of the angular momentum and the complete Hamiltonian

$$
\hat{H}=\frac{1}{2}\left(\frac{\hat{L}_{x}^{2}}{I_{1}}+\frac{\hat{L}_{y}^{2}}{I_{2}}+\frac{\hat{L}_{z}^{2}}{I_{3}}\right)
$$

in the body-fixed frame with principal axes, involving the moments of inertia $I_{1} \leq I_{2} \leq I_{3}$. The separated equations are of the Lamé type, and its polynomial solutions are exact in principle, but could not be numerically implemented for higher excited states at that time. Consequently, the study of the rotations of asymmetric molecules developed along the perturbation theory route taking the symmetric prolate and oblate molecule spherical harmonic solutions as the non-perturbed starting point [7, 8, 16, 29, 35].

Patera and Winternitz used an alternative energy operator

$$
E=-4\left(\hat{L}_{1}^{2}+r \hat{L}_{2}^{2}\right), \quad 0<r<1,
$$

in their work [24]. On the other hand, Piña [27] and Valdéz and Piña [32] used the alternative parametrization of the original Hamiltonian of equation (1), in the form

$$
\hat{H}=\frac{1}{2} Q \hat{L}^{2}+\frac{1}{2} P\left(e_{1} \hat{L}_{x}^{2}+e_{2} \hat{L}_{y}^{2}+e_{3} \hat{L}_{z}^{2}\right),
$$

where

$$
Q=\frac{1}{3}\left(\frac{1}{I_{1}}+\frac{1}{I_{2}}+\frac{1}{I_{3}}\right)
$$


is the average of the three inverses of the moments of inertia characterizing a spherical top,

$$
P^{2}=\frac{2}{9}\left[\left(\frac{1}{I_{1}}-\frac{1}{I_{2}}\right)^{2}+\left(\frac{1}{I_{1}}-\frac{1}{I_{3}}\right)^{2}+\left(\frac{1}{I_{2}}-\frac{1}{I_{3}}\right)^{2}\right]
$$

measures the magnitude of the asymmetry of the molecule and

$$
\hat{H}^{*}=\frac{1}{2}\left(e_{1} L_{x}^{2}+e_{2} L_{y}^{2}+e_{3} L_{z}^{2}\right)
$$

is the asymmetry distribution Hamiltonian, with parameters $e_{1}, e_{1}$ and $e_{3}$ such that

$$
e_{1} \geq e_{2} \geq e_{3}, \quad e_{1}+e_{2}+e_{3}=0, \quad e_{1}^{2}+e_{2}^{2}+e_{3}^{2}=\frac{3}{2} .
$$

The inequalities follow from those for the moments of inertia, the vanishing of their sum reflects the zero trace of the matrix of the inverses of the moments of inertia after the term in $Q$ is separated, and the coefficient in $P^{2}$ and the sums of the squares are correlated. Only one of the parameters $e_{i}$ is independent, the set of $I_{1}, I_{2}, I_{3}$ being replaced by $Q, P, e_{i}$.

Both of our works $[9,10]$ used the parametrizations of equations (2) and (3) with a single independent parameter $e_{i}$, in analogy with that in equation (1). At that time we were not aware of [24]. Not surprisingly, their equivalence can be established and our respective methodologies also overlap. In fact, our solution using the spherical canonical bases to construct the matrix representation of $\hat{H}^{*}$ and determine its eigenvalues and eigenfunctions by diagonalization [10] is related to Sections 2 and 3 in [24]. On the other hand, our construction of the Lamé eigenvalues and polynomials also by diagonalization of the matrices representing the recurrence relations for the series expansions in the Jacobi elliptic function representation of the spheroconal coordinates [10], has as well points of contact with parts of Section 6 in [24]. We have just become aware of some additional references $[13,15]$ and [14] of that period, whose titles describe the overlap with this contribution. Specifically, the role of the $D_{2}$ group of rotations by $\pi$ around the cartesian coordinate axes as a finite symmetry group is explicitly recognized in [25] as the missing ingredient for the complete characterization of the different species of Lamé spheroconal harmonics.

We refer the reader to $[10,27,32]$ for the details or equations in what follows. The transformation equations between spheroconal and cartesian coordinates are

$$
\begin{aligned}
& x=r \operatorname{dn}\left(\chi_{1} \mid k_{1}^{2}\right) \operatorname{sn}\left(\chi_{2} \mid k_{2}^{2}\right), \quad y=r \operatorname{cn}\left(\chi_{1} \mid k_{1}^{2}\right) \operatorname{cn}\left(\chi_{2} \mid k_{2}^{2}\right), \\
& z=r \operatorname{sn}\left(\chi_{1} \mid k_{1}^{2}\right) \operatorname{dn}\left(\chi_{2} \mid k_{2}^{2}\right)
\end{aligned}
$$

in terms of Jacobi elliptic functions sn $\left(\chi \mid k_{i}^{2}\right)$, cn $\left(\chi \mid k_{i}^{2}\right)$ and $\operatorname{dn}\left(\chi \mid k_{i}^{2}\right)$ with matching parameters $k_{1}^{2}+k_{2}^{2}=1[1,20]$. The elliptic functions are related as

$$
\operatorname{cn}^{2}\left(\chi \mid k^{2}\right)=1-\operatorname{sn}^{2}\left(\chi \mid k^{2}\right), \quad \operatorname{dn}^{2}\left(\chi \mid k^{2}\right)=1-k^{2} \operatorname{sn}^{2}\left(\chi \mid k^{2}\right),
$$

and their derivatives are

$$
\begin{aligned}
& \frac{d}{d \chi} \operatorname{sn}\left(\chi \mid k^{2}\right)=\operatorname{cn}\left(\chi \mid k^{2}\right) \operatorname{dn}\left(\chi \mid k^{2}\right), \quad \frac{d}{d \chi} \operatorname{cn}\left(\chi \mid k^{2}\right)=-\operatorname{sn}\left(\chi \mid k^{2}\right) \operatorname{dn}\left(\chi \mid k^{2}\right), \\
& \frac{d}{d \chi} \operatorname{dn}\left(\chi \mid k^{2}\right)=-k^{2} \operatorname{sn}\left(\chi \mid k^{2}\right) \operatorname{cn}\left(\chi \mid k^{2}\right) .
\end{aligned}
$$

The transformation equations (4) and equations (5) allow the identification of $r$ as the radial spherical coordinate, $\chi_{1}$ as an elliptical cone coordinate with axis along the $x$-axis and $\chi_{2}$ as an elliptical cone coordinate with axis along $z$-axis. Their respective domains are $-\pi<\operatorname{am}\left(\chi_{i}\right)<\pi$ 
and $-\pi / 2<\operatorname{am}\left(\chi_{j}\right)<\pi / 2$ for $k_{i}^{2}>k_{j}^{2}$. Since $k_{1}^{2}$ is always associated with variable $\chi_{1}$ and $k_{2}^{2}$ with $\chi_{2}$, from now on in this manuscript, we simplify the notation as $\left(\chi_{i} \mid k_{i}^{2}\right) \rightarrow\left(\chi_{i}\right)$, for the sake of space saving.

The transformation equations (4) and the equations (6) lead in turn to the scale factors

$$
h_{r}=1, \quad h_{\chi}=h_{\chi_{1}}=h_{\chi_{2}}=r \sqrt{1-k_{1}^{2} \operatorname{sn}^{2}\left(\chi_{1}\right)-k_{2}^{2} \operatorname{sn}^{2}\left(\chi_{2}\right)}
$$

and to the Laplace operator

$$
\nabla^{2}=\frac{1}{r^{2}} \frac{\partial}{\partial r}\left(r^{2} \frac{\partial}{\partial r}\right)+\frac{1}{h_{\chi}^{2}}\left(\frac{\partial^{2}}{\partial \chi_{1}^{2}}+\frac{\partial^{2}}{\partial \chi_{2}^{2}}\right)
$$

From the latter the identification of the square of the angular momentum is immediate:

$$
\hat{L}^{2}=-\frac{\hbar^{2} r^{2}}{h_{\chi}^{2}}\left(\frac{\partial^{2}}{\partial \chi_{1}^{2}}+\frac{\partial^{2}}{\partial \chi_{2}^{2}}\right) .
$$

The cartesian components of the angular momentum vector itself can be found in the Appendix of [10], and allow the construction of the explicit form of the asymmetry distribution Hamiltonian

$$
\hat{H}^{*}=-\frac{\hbar^{2} r^{2}}{2 h_{\chi}^{2}}\left(\left(e_{1}-\left(e_{1}-e_{2}\right) \operatorname{sn}^{2}\left(\chi_{2}\right)\right) \frac{\partial^{2}}{\partial \chi_{1}^{2}}+\left(e_{3}-\left(e_{2}-e_{3}\right) \operatorname{sn}^{2}\left(\chi_{2}\right)\right) \frac{\partial^{2}}{\partial \chi_{2}^{2}}\right) .
$$

Since $\hat{L}^{2}$ commutes with $\hat{H}^{*}$, as easily tested using equation (2), both operators admit common eigenfunctions with the factorizable form

$$
\Psi\left(\chi_{1}, \chi_{2}\right)=\Lambda\left(\chi_{1}\right) \Lambda\left(\chi_{2}\right)
$$

for which the eigenvalue equations of the operators of equation (8) and (9), with eigenvalues $\ell(\ell+1)$ and $E^{*}$ become separable. Indeed by using the angular scale factors of equation $(7)$, the separability can be accomplished provided the geometric parameters in equations (4) and the dynamic parameters in equation (9) are connected by

$$
k_{1}^{2}=\frac{e_{2}-e_{3}}{e_{1}-e_{3}}, \quad k_{2}^{2}=\frac{e_{1}-e_{2}}{e_{1}-e_{3}} .
$$

This means that the specific spheroconal coordinate system is tailored to the asymmetry distribution of the molecule.

The next result is that each of the factors in equation (10) satisfies the Lamé equation $[21,33,34]$

$$
\left[-\frac{d^{2}}{d \chi_{i}^{2}}+\ell(\ell+1) k_{i}^{2} \operatorname{sn}^{2}\left(\chi_{i}\right)\right] \Lambda_{i}\left(\chi_{i}\right)=h_{i} \Lambda_{i}\left(\chi_{i}\right)
$$

for $i=1,2$, with the respective eigenvalues

$$
h_{1}=-\frac{\ell(\ell+1) e_{3}}{e_{1}-e_{3}}+\frac{2 E^{*}}{e_{1}-e_{3}}, \quad h_{2}=\frac{\ell(\ell+1) e_{1}}{e_{1}-e_{3}}-\frac{2 E^{*}}{e_{1}-e_{3}} .
$$

Their combinations

$$
h_{1}+h_{2}=\ell(\ell+1), \quad e_{1} h_{1}+e_{3} h_{2}=2 E^{*},
$$

provide the eigenvalues of $\hat{L}^{2}$ and $\hat{H}^{*}$, respectively. 
Table 1. Matching Lamé polynomials according to species and parities.

\begin{tabular}{c|cccccccc}
$\mathrm{A}$ & 1 & $d$ & $c$ & $s$ & $d c$ & $d s$ & $c s$ & $d c s$ \\
$\mathrm{~B}$ & 1 & $s$ & $c$ & $d$ & $s c$ & $s d$ & $c d$ & $s c d$ \\
\hline $\mathrm{AB}$ & 1 & $x$ & $y$ & $z$ & $x y$ & $x z$ & $y z$ & $x y z$ \\
\hline$\Pi_{x}$ & + & - & + & + & - & - & + & - \\
$\Pi_{y}$ & + & + & - & + & - & + & - & - \\
$\Pi_{z}$ & + & + & + & - & + & - & - & -
\end{tabular}

Next, the solutions of the Lamé equation (11) are also reviewed. The derivative equations (6) for the elliptic functions, represented by their initial letters allow the identification of $1, s, c$, $d, d c, d s, s c, d c s$ as removable singularity factors in the differential equation. The dependence of both operators $\hat{L}^{2}$ and $\hat{H}^{*}$ on the squares of $\hat{L}_{x}, \hat{L}_{y}$ and $\hat{L}_{z}$ guarantee that their common eigenfunctions, equation (10), have definite inversion parities under $x \rightarrow-x, y \rightarrow-y, z \rightarrow-z$. Correspondingly, the Lamé functions get classified into two kinds, each with four species sharing the same number of even or odd factors in the removable singularity factors. The Lamé functions in the respective elliptic cone coordinates $\chi_{1}$ and $\chi_{2}$ in equation (10) must be matched according to their singularity factors $A\left(\chi_{1}\right)$ and $B\left(\chi_{2}\right)$, and the respective parities from equations (4), as illustrated in Table 1.

There are two kinds of Lamé spheroconal harmonic functions distinguished by their overall parity $(-)^{\ell}=\Pi_{x} \Pi_{y} \Pi_{z}$. For each value of $\ell$ each kind may contain four different species characterized by the respective singularity factors.

The solutions of the Lamé differential equation (11) have the general form

$$
\Lambda^{A}\left(\chi_{i}\right)=A\left(\chi_{i}\right) \sum_{s=0}^{N_{\max }^{A}} a_{s}^{A} \operatorname{sn}^{2 s}\left(\chi_{i}\right)
$$

as a series of even-power of the elliptical $\operatorname{sn}\left(\chi_{i}\right)$ function, where the expansion coefficients satisfy three-term recurrence relations, which the interested reader may see in equations (32)(39) of [10], for the eight different species $A$. For the lower $\ell$ eigenstates the eigenvalues $h^{A}\left(k_{i}^{2}\right)$ and ratios of the expansion coefficients $a_{s}^{A} / a_{0}^{A}$ can be evaluated in a straightforward way from equation (11), and the linear independence of the powers of $\operatorname{sn}\left(\chi_{i}\right)$. The corresponding results are illustrated in Table 2 for the individual Lamé polynomials and in Table 3 for the matched spheroconal harmonics.

For higher values of $\ell$ it is more practical to cast the recurrence relations into eigenvalue problem matrix forms and to obtain from their diagonalizations the respective eigenvalues and expansion coefficients. The sizes of the matrices are as follows for $\ell$ even:

$$
N_{\max }^{1}=\frac{\ell}{2}+1 \quad \text { and } \quad N_{\max }^{s c}=N_{\max }^{s d}=N_{\max }^{c d}=\frac{\ell}{2},
$$

and for $\ell$ odd:

$$
N_{\max }^{s}=N_{\max }^{c}=N_{\max }^{d}=\frac{\ell+1}{2} \quad \text { and } \quad N_{\max }^{s c d}=\frac{\ell-1}{2} .
$$

In both cases the total dimensions are $2 \ell+1$, corresponding to the number of independent spheroconal harmonic polynomials for each value of $\ell$. For each species there are $N_{\max }^{A}$ different eigenvalues which can be ordered according to their increasing values

$$
h_{1}^{A}<h_{2}^{A}<h_{3}^{A}<\cdots<h_{i}^{A}<\cdots<h_{N_{\max }^{A}-1}^{A}<h_{N_{\max }^{A}}^{A},
$$


or their decreasing values

$$
h_{N_{\max }}^{B}>h_{N_{\max }-1}^{B}>\cdots>h_{j}^{B}>\cdots>h_{3}^{B}>h_{2}^{B}>h_{1}^{B} .
$$

Obviously, the expansion coefficients in equation (14) depend on the specific eigenvalues $a_{s}\left(h_{i}^{A}\right)$, $a_{s}\left(h_{j}^{B}\right)$. The first of equations (13) suggests the matching of the smaller eigenvalues of one species with the larger ones of the companion species for the construction of the complete set of spheroconal harmonics $\ell A B i j$.

Let us also analyze the number of nodes in the respective factors of Lamé functions in equation (14), $n^{A}$ and $n^{p}$. For the singularity removing factors their specific values are as follows:

$$
\begin{array}{ll}
n^{1}=n^{d}=0, & n^{c}=0 \text { or } 1, \quad n^{s}=1, \\
n^{d c}=0 \text { or } 1, & n^{d s}=1, \quad n^{c s}=1 \text { or } 2, \quad n^{d c s}=1 \text { or } 2 .
\end{array}
$$

The polynomial of degree $2 N_{\max }^{A}$ in $\operatorname{sn}(\chi)$ has at the most $N_{\max }^{A}$ zeros $\operatorname{sn}^{2}\left(\chi_{p}\right)$, appearing by pairs with opposite signs $\pm \operatorname{sn}\left(\chi_{p}\right)$ with the possible numbers

$$
n^{p}=0,2,4, \ldots, 2\left(N_{\max }-1\right)
$$

counting the symmetric sheets of the nodal elliptical cones.

Therefore, the total number of nodes for $\Lambda_{n_{1}}^{\ell A}\left(\chi_{1}\right)$ Lamé polynomial is

$$
n_{1}=n^{A}+n^{p}=0,1,2, \ldots, \ell
$$

and likewise for its matching companion $\Lambda_{n_{2}}^{\ell B}\left(\chi_{2}\right)$

$$
n_{2}=n^{B}+n^{p}=0,1,2, \ldots, \ell .
$$

Correspondingly, the spheroconal harmonic functions from the product of the pairs of matched Lamé polynomials exhibit the possible numbers

$$
n^{A}+n^{B}=0,1,2,3
$$

counting the shared nodal cartesian planes, as limiting cases of nodal elliptical cones, for the respective species

$$
A B=1 ; x, y, z ; x y, x z, y z ; x y z .
$$

In turn,

$$
n_{1}+n_{2}=\ell
$$

counts the total number of nodes characterizing any harmonic function of order $\ell$.

The ordering indices $i, j$ for the eigenvalues in equations (15) and (16) can be replaced with the quantum numbers $n_{1}$ and $n_{2}$. This is completely justified by recognizing that the eigenvalues with matching quantum numbers, according to equation (22), are also matched to satisfy

$$
h_{n_{1}}^{\ell A}+h_{n_{2}}^{\ell B}=\ell(\ell+1)
$$

as required by equation (13).

Table 2 illustrates the eigenvalues $h_{n_{i}}^{\ell A}$ of the Lamé functions for the successive values of $\ell=0,1,2, \ldots$, and the respective species with the singularity removing factors. The eigenvalues and their quantum numbers $n_{1}=0,1,2, \ldots$ are listed in increasing order. The associated polynomial factors in the eigenfunctions, equation (14), are also included.

Table 3 illustrates the matching of Lamé eigenvalues and polynomials to obtain the spheroconal harmonics $\ell A B n_{1} n_{2}$ and their eigenenergies $2 E^{*}$ from equation (13), written in terms of the asymmetry distribution parameter $e_{i}$. The readers may check the ordering of the individual Lamé eigenvalues, correlated with the increasing of the number of nodes in the polynomials in Table 2. The matching according to equations (22) and (23), and the ordering of increasing energies can be appreciated in Table 3. 
Table 2. Lamé eigenvalues $h_{n_{1}}^{\ell A}$ and polynomials.

\begin{tabular}{|c|c|c|c|}
\hline & $A$ & $h_{n_{i}}^{A}$ & Polynomial \\
\hline$\ell=0$ & 1 & $h_{0}^{1}=0$ & 1 \\
\hline$\ell=1$ & $\begin{array}{l}d \\
c \\
s\end{array}$ & $\begin{aligned} h_{0}^{d} & =k_{i}^{2} \\
h_{1}^{c} & =1 \\
h_{1}^{s} & =1+k_{i}^{2}\end{aligned}$ & $\begin{array}{l}1 \\
1 \\
1\end{array}$ \\
\hline$\ell=2$ & $\begin{array}{l}d c \\
d s \\
c s \\
1 \\
1\end{array}$ & $\begin{array}{l}h_{1}^{d c}=1+k_{i}^{2} \\
h_{1}^{d s}=1+4 k_{i}^{2} \\
h_{2}^{c s}=4+k_{i}^{2} \\
h_{0}^{1}=2\left(1+k_{i}^{2}\right)-2 \sqrt{1-k_{i}^{2}\left(1-k_{i}^{2}\right)} \\
h_{2}^{1}=2\left(1+k_{i}^{2}\right)+2 \sqrt{1-k_{i}^{2}\left(1-k_{i}^{2}\right)}\end{array}$ & $\begin{array}{l}1 \\
1 \\
1 \\
1-\frac{h_{0}^{1}}{2} \operatorname{sn}^{2}\left(\chi_{i}\right) \\
1-\frac{h_{2}^{1}}{2} \operatorname{sn}^{2}\left(\chi_{i}\right)\end{array}$ \\
\hline$\ell=3$ & $\begin{array}{l}d c s \\
d \\
d \\
c \\
c \\
s \\
s\end{array}$ & $\begin{array}{l}h_{2}^{d c s}=4\left(1+k_{i}^{2}\right) \\
h_{0}^{d}=\left(5 k_{i}^{2}+2\right)-2 \sqrt{4 k_{i}^{4}-k_{i}^{2}+1} \\
h_{2}^{d}=\left(5 k_{i}^{2}+2\right)+2 \sqrt{4 k_{i}^{4}-k_{i}^{2}+1} \\
h_{1}^{c}=\left(5+2 k_{i}^{2}\right)-2 \sqrt{4-k_{i}^{2}\left(1-k_{i}^{2}\right)} \\
h_{3}^{c}=\left(5+2 k_{i}^{2}\right)+2 \sqrt{4-k_{i}^{2}\left(1-k_{i}^{2}\right)} \\
h_{1}^{s}=5\left(1+k_{i}^{2}\right)-2 \sqrt{4 k_{i}^{4}-7 k_{i}^{2}+4} \\
h_{3}^{s}=5\left(1+k_{i}^{2}\right)+2 \sqrt{4 k_{i}^{4}-7 k_{i}^{2}+4}\end{array}$ & $\begin{array}{l}1 \\
1+\frac{k_{i}^{2}-h_{0}^{d}}{2} \operatorname{sn}^{2}\left(\chi_{i}\right) \\
1+\frac{k_{i}^{2}-h_{2}^{d}}{2} \operatorname{sn}^{2}\left(\chi_{i}\right) \\
1+\frac{1-h_{1}^{c}}{2} \operatorname{sn}^{2}\left(\chi_{i}\right) \\
1+\frac{1-h_{3}^{c}}{2} \operatorname{sn}^{2}\left(\chi_{i}\right) \\
1+\frac{1+k_{i}^{2}-h_{1}^{s}}{6} \operatorname{sn}^{2}\left(\chi_{i}\right) \\
1+\frac{1+k_{i}^{2}-h_{3}^{s}}{6} \operatorname{sn}^{2}\left(\chi_{i}\right)\end{array}$ \\
\hline$\ell=4$ & $\begin{array}{l}d c \\
d c \\
d s \\
d s \\
c s \\
c s\end{array}$ & $\begin{array}{c}h^{3}-20\left(1+k_{i}^{2}\right) h^{2} \\
+\left[64\left(1+k_{i}^{2}\right)+208 k_{i}^{2}\right] h \\
-640 k_{i}^{2}\left(1+k_{i}^{2}\right)=0 \\
h_{1}^{d c}=5\left(1+k_{i}^{2}\right)-2 \sqrt{4+k_{i}^{2}+4 k_{i}^{4}} \\
h_{3}^{d c}=5\left(1+k_{i}^{2}\right)+2 \sqrt{4+k_{i}^{2}+4 k_{i}^{4}} \\
\left.h_{1}^{d s}=5\left(1+2 k_{i}^{2}\right)-2 \sqrt{4-9 k_{i}^{2}\left(1-k_{i}^{2}\right.}\right) \\
\left.h_{3}^{d s}=5\left(1+2 k_{i}^{2}\right)+2 \sqrt{4-9 k_{i}^{2}\left(1-k_{i}^{2}\right.}\right) \\
h_{2}^{c s}=5\left(2+k_{i}^{2}\right)-2 \sqrt{9-9 k_{i}^{2}+4 k_{i}^{4}} \\
h_{4}^{c s}=5\left(2+k_{i}^{2}\right)+2 \sqrt{9-9 k_{i}^{2}+4 k_{i}^{4}}\end{array}$ & $\begin{array}{l}1+\frac{a_{1}}{a_{0}} \operatorname{sn}^{2}\left(\chi_{i}\right)+\frac{a_{2}}{a_{0}} \operatorname{sn}^{4}\left(\chi_{i}\right) \\
a_{1}=-\frac{h_{n_{i}}^{1}}{2} a_{0} ; a_{2}=\frac{7 k_{i}^{2} h_{n_{i}}^{1}}{16\left(1+k_{i}^{2}\right)-h_{n_{i}}^{1}} a_{0} \\
\text { The roots of the polynomial } \\
\text { are } h_{0}^{1}, h_{2}^{1}, h_{4}^{1} \\
1-\frac{h_{1}^{d c}-\left(1+k_{i}^{2}\right)}{2} \operatorname{sn}^{2}\left(\chi_{i}\right) \\
1-\frac{h_{3}^{d c}-\left(1+k_{i}^{2}\right)}{2} \operatorname{sn}^{2}\left(\chi_{i}\right) \\
1-\frac{h_{1}^{d s}-\left(1+4 k_{i}^{2}\right)}{6} \operatorname{sn}^{2}\left(\chi_{i}\right) \\
1-\frac{h_{3}^{d s}-\left(1+4 k_{i}^{2}\right)}{6} \operatorname{sn}^{2}\left(\chi_{i}\right) \\
1-\frac{h_{2}^{c s}-\left(4+k_{i}^{2}\right)}{6} \operatorname{sn}^{2}\left(\chi_{i}\right) \\
1-\frac{h_{4}^{c s}-\left(4+k_{i}^{2}\right)}{6} \operatorname{sn}^{2}\left(\chi_{i}\right)\end{array}$ \\
\hline
\end{tabular}

\section{Raising and lowering of quantum numbers $n_{1}$ and $n_{2}$ for spheroconal harmonic polynomials of a chosen kind and species $\ell A B n_{1} n_{2}$}

The spheroconal harmonic polynomials with their labels for angular momentum $\ell$, species $A B$ and number of nodes $n_{1}$ and $n_{2}$ can be written as the products of the pairs of matching Lamé polynomials

$$
\begin{aligned}
& \Psi_{n_{1} n_{2}}^{\ell A B}\left(\chi_{1}, \chi_{2} ; h_{n_{1}}^{\ell A}, h_{n_{2}}^{\ell B}\right)=\Lambda_{n_{1}}^{\ell A}\left(\chi_{1}, h_{n_{1}}^{\ell A}\right) \Lambda_{n_{2}}^{\ell B}\left(\chi_{2}, h_{n_{2}}^{\ell B}\right), \\
& \Lambda_{n_{1}}^{\ell A}\left(\chi_{1}, h_{n_{1}}^{\ell A}\right)=A\left(\chi_{1}\right) a_{0}^{A}\left(h_{n_{1}}^{\ell A}\right) \sum_{s=0}^{N_{\max }} \bar{a}_{s}^{A}\left(h_{n_{1}}^{\ell A}\right) \operatorname{sn}^{2 s}\left(\chi_{1}\right)
\end{aligned}
$$


Table 3. Eigenenergies $2 E^{*}$ in units of $\hbar^{2}$ for Lamé spheroconal harmonics $\ell A B n_{1} n_{2}$.

\begin{tabular}{l|ll|ll|l} 
& $A$ & $B$ & $n_{1}$ & $n_{2}$ & $2 E^{*}$ \\
\hline \hline$\ell=0$ & 1 & 1 & 0 & 0 & 0 \\
\hline$\ell=1$ & $d$ & $s$ & 0 & 1 & $-e_{1}$ \\
& $c$ & $c$ & 1 & 0 & $-e_{2}$ \\
\hline$\ell=2$ & $s$ & $d$ & 1 & 0 & $-e_{3}$ \\
\hline$\ell=3$ & 1 & 0 & 2 & -3 \\
& $d c$ & $s c$ & 1 & 1 & $3 e_{3}$ \\
& $d s$ & $s d$ & 1 & 1 & $3 e_{2}$ \\
& $c s$ & $c d$ & 2 & 0 & $3 e_{1}$ \\
& 1 & 1 & 2 & 0 & 3 \\
\hline$\ell=4$ & $s$ & 0 & 3 & $-3 e_{1}-3 \sqrt{5-4 e_{1}^{2}}$ \\
& $c$ & $c$ & 1 & 2 & $-3 e_{2}-3 \sqrt{5-4 e_{2}^{2}}$ \\
& $s$ & $d$ & 1 & 2 & $-3 e_{3}-3 \sqrt{5-4 e_{3}^{2}}$ \\
& $d c s$ & $s c d$ & 2 & 1 & 0 \\
& $d$ & $s$ & 2 & 1 & $-3 e_{1}+3 \sqrt{5-4 e_{1}^{2}}$ \\
& $c$ & $c$ & 3 & 0 & $-3 e_{2}+3 \sqrt{5-4 e_{2}^{2}}$ \\
& $s$ & $d$ & 3 & 0 & $-3 e_{3}+3 \sqrt{5-4 e_{3}^{2}}$ \\
\hline$\ell$ & 1 & 0 & 4 & $e_{1} h_{0}^{1}\left(k_{1}^{2}\right)+e_{3} h_{4}^{1}\left(k_{2}^{2}\right)$ \\
& $d c$ & $s c$ & 1 & 3 & $5 e_{3}-\sqrt{21+60 e_{3}^{2}}$ \\
& $d s$ & $s d$ & 1 & 3 & $5 e_{2}-\sqrt{21+60 e_{2}^{2}}$ \\
& $c s$ & $c d$ & 2 & 2 & $5 e_{1}-\sqrt{21+60 e_{1}^{2}}$ \\
& 1 & 1 & 2 & 2 & $e_{1} h_{2}^{1}\left(k_{1}^{2}\right)+e_{3} h_{2}^{1}\left(k_{2}^{2}\right)$ \\
& $d c$ & $s c$ & 3 & 1 & $5 e_{3}+\sqrt{21+60 e_{3}^{2}}$ \\
& $d s$ & $s d$ & 3 & 1 & $5 e_{2}+\sqrt{21+60 e_{2}^{2}}$ \\
& $c s$ & $c d$ & 4 & 0 & $5 e_{1}+\sqrt{21+60 e_{1}^{2}}$ \\
& 1 & 1 & 4 & 0 & $e_{1} h_{4}^{1}\left(k_{1}^{2}\right)+e_{3} h_{0}^{1}\left(k_{2}^{2}\right)$
\end{tabular}

factorizing the zero power coefficients $a_{0}^{A}\left(h_{n_{1}}^{\ell A}\right)$ and using the ratio of coefficients for the other powers

$$
\bar{a}_{s}^{A}\left(h_{n_{1}}^{\ell A}\right)=\frac{a_{s}^{A}\left(h_{n_{1}}^{\ell A}\right)}{a_{0}^{A}\left(h_{n_{1}}^{\ell A}\right)}
$$

and similarly for its companion with $A \rightarrow B$ and $n_{1} \rightarrow n_{2}$. As established in the previous section, equations (22) and (23) define the matching of quantum numbers and eigenvalues, respectively.

For chosen values of $\ell, A$ and $B$, the removed singularity nodes are fixed in numbers $n^{A}$ and $n^{B}$, according to equations (17)-(21). Then according to equations (18)-(20) the number of nodes $n^{p}, n_{1}$ and $n_{2}$ change in steps of 2 . the inequalities in equations (15) and (16) for the increasing and decreasing eigenvalues become

$$
h_{n^{A}}^{\ell A}<h_{n^{A}+2}^{\ell A}<\cdots<h_{n_{1}-2}^{\ell A}<h_{n_{1}}^{\ell A}<h_{n_{1}+2}^{\ell A}<\cdots<h_{\ell-n^{B}-2}^{\ell A}<h_{\ell-n^{B}}^{\ell A},
$$

and

$$
h_{\ell-n^{A}}^{\ell B}>h_{\ell-n^{A}-2}^{\ell B}>\cdots>h_{n_{2}+2}^{\ell B}>h_{n_{2}}^{\ell B}>h_{n_{2}-2}^{\ell B}>\cdots>h_{n^{B}+2}^{\ell B}>h_{n^{B}}^{\ell B} .
$$

If we start from the state $\ell A B n_{1} n_{2}$ of equation (24) depending on the eigenvalues appearing in the middle of equations (27) and (28), the state involving their neighbors to the right have 
raised $n_{1}$ by two units and lowered $n_{2}$ by two units with the corresponding change in the eigenvalues which remain satisfying matching conditions of equations (22) and (23); the same changes operate in equations (25) and (26) for the eigenfunctions via the eigenvalue dependence of the expansion coefficients. Similarly, if we had moved to the neighbors on the left: $n_{1} \rightarrow n_{1}-2$ and $n_{2} \rightarrow n_{1}+2$ with the complementary lowering and raising by two, with their consequences in the changes in the matching eigenvalues and the other state of the same species. Obviously, the lowering and raising actions can be extended to the right and left ends of equations (27) and (28) covering all the $N_{\max }^{A}=N_{\max }^{B}$ states of the $\ell A B$ species. Examples of these connections can be found in Tables 2 and 3.

As a prelude to the following two sections we include a couple of purely spheroconal coordinate tools. The first one illustrates the successive species changing actions of the derivative operator on the singularity removing factors and even-power polynomials appearing in the process:

\begin{tabular}{l|l|l} 
& $\frac{\partial}{\partial \chi_{i}}$ & $\frac{\partial^{2}}{\partial \chi_{i}^{2}}$ \\
\hline \hline 1 & 0 & 0 \\
$s$ & $c d$ & $\left(-k_{i}^{2} c^{2}-d^{2}\right) s$ \\
$c$ & $-s d$ & $\left(-k_{i}^{2} s^{2}+d^{2}\right) c$ \\
$d$ & $-k_{i}^{2} s c$ & $-k_{i}^{2}\left(c^{2}-s^{2}\right) d$ \\
$c d$ & $\left(-k_{i}^{2} c^{2}-d^{2}\right) s$ & $\left(-k_{i}^{2} c^{2}-d^{2}+2 k_{i}^{2} s^{2}\right) c d$ \\
$s d$ & $\left(-k_{i}^{2} s^{2}+c^{2}\right) c$ & $\left(k_{i}^{2} s^{2}-d^{2}-2 k_{i}^{2} c^{2}\right) s d$ \\
$s c$ & $\left(s^{2}-c^{2}\right) d$ & $\left(-k_{i}^{2} s^{2}+k_{i}^{2} c^{2}+2 k_{i}^{2} d^{2}\right) s c$ \\
$s c d$ & $-k^{2} c^{2} s^{2}-d^{2} s^{2}+d^{2} c^{2}$ & $2\left[k_{i}^{2}\left(s^{2}-c^{2}\right)+\left(k_{i}^{2} s^{2}-d^{2}\right)-\left(k_{i}^{2} c^{2}+d^{2}\right)\right] s c d$ \\
$s^{2}, c^{2}, d^{2}$ & $2 s c d\left(1,-1,-k_{i}^{2}\right)$ &
\end{tabular}

The first derivative causes changes of species with one factor to species with the other two factors and viceversa. The species 1 is represented by the top entry, and also by the bottom one; and species $d c s$ and 1 are also connected by their first derivatives as illustrated by the two bottom entries. The second derivative returns each species to the original one. The even-power polynomials can be reduced to polynomials in $\operatorname{sn}^{2}(\chi)$ via equation (5).

The second tool is connected with equation (25), expressing the set of Lamé polynomials $\ell A n_{1}$ as a linear combination of the $A(\chi) \operatorname{sn}^{2 s}(\chi)$ basis. Both bases have the same dimension $N_{\max }^{A}+1$, and the members of the latter can be expressed as linear combinations of Lamé polynomials. The transformation coefficients $\bar{a}_{s n_{i}}^{A \text { inv }}$ are the elements of the inverse matrix of $\bar{a}_{s}^{A}\left(h_{n_{1}}^{\ell A}\right)$.

\section{Angular momentum cartesian components connecting pairs of polynomials of the four different species with a common eigenvalue $\ell$}

The explicit expressions for the cartesian components of the angular momentum operators in spheroconal coordinates are borrowed as equations (A15)-(A17) in [10]:

$$
\begin{aligned}
& \hat{L}_{x}=\frac{-i \hbar r^{2}}{h_{\chi}^{2}}\left[\operatorname{dn}\left(\chi_{1}\right) \operatorname{cn}\left(\chi_{2}\right) \operatorname{dn}\left(\chi_{2}\right) \frac{\partial}{\partial \chi_{1}}+k_{1}^{2} \operatorname{sn}\left(\chi_{1}\right) \operatorname{cn}\left(\chi_{1}\right) \operatorname{sn}\left(\chi_{2}\right) \frac{\partial}{\partial \chi_{2}}\right] \\
& \hat{L}_{y}=\frac{-i \hbar r^{2}}{h_{\chi}^{2}}\left[-\operatorname{cn}\left(\chi_{1}\right) \operatorname{sn}\left(\chi_{2}\right) \operatorname{dn}\left(\chi_{2}\right) \frac{\partial}{\partial \chi_{1}}+\operatorname{sn}\left(\chi_{1}\right) \operatorname{dn}\left(\chi_{1}\right) \operatorname{cn}\left(\chi_{2}\right) \frac{\partial}{\partial \chi_{2}}\right] \\
& \hat{L}_{z}=\frac{-i \hbar r^{2}}{h_{\chi}^{2}}\left[-k_{2}^{2} \operatorname{sn}\left(\chi_{1}\right) \operatorname{sn}\left(\chi_{2}\right) \operatorname{cn}\left(\chi_{2}\right) \frac{\partial}{\partial \chi_{1}}-\operatorname{cn}\left(\chi_{1}\right) \operatorname{dn}\left(\chi_{1}\right) \operatorname{dn}\left(\chi_{2}\right) \frac{\partial}{\partial \chi_{2}}\right] .
\end{aligned}
$$


Table 4. Eigenfunctions with $\ell=1$ and its resulting application of each cartesian angular momentum operator.

\begin{tabular}{c|ccc} 
& $\hat{L}_{x}$ & $\hat{L}_{y}$ & $\hat{L}_{z}$ \\
\hline \hline$\Lambda_{0}^{d} \Lambda_{1}^{s}$ & 0 & $-\Lambda_{1}^{s} \Lambda_{0}^{d}$ & $\Lambda_{1}^{c} \Lambda_{0}^{c}$ \\
$\Lambda_{1}^{c} \Lambda_{0}^{c}$ & $\Lambda_{1}^{s} \Lambda_{0}^{d}$ & 0 & $-\Lambda_{0}^{d} \Lambda_{1}^{s}$ \\
$\Lambda_{1}^{s} \Lambda_{0}^{d}$ & $-\Lambda_{1}^{c} \Lambda_{0}^{c}$ & $\Lambda_{0}^{d} \Lambda_{1}^{s}$ & 0
\end{tabular}

This section evaluates the actions of these operators on the lower $\ell A B n_{1} n_{2}$ spheroconal harmonic polynomials of the same kind for a fixed value of $\ell$, connecting with different species $\ell A^{\prime} B^{\prime} n_{1}^{\prime} n_{2}^{\prime}$.

The calculations are straightforward requiring care, labor and some guidelines. The derivatives $\partial / \partial \chi_{i}$ act only on the first or second factor in equation (24) for $i=1$ or 2 . The individual changes of species were already described in the first tool of the prelude, and for an initial matching species $A B$ the final ones $A^{\prime} B^{\prime}$ follow. Notice the appearance of the square of the angular scale factor in the three operators in equations (29)-(31). This requires that the application of the operators inside the brackets to the spheroconal harmonic polynomials provides the $A^{\prime} B^{\prime}$ factor and also the $h_{\chi}^{2} / r^{2}$ factor to compensate the one in the previous line. This requirement is indeed satisfied. The remaining factor can be reduced to a polynomial in even-powers of $\operatorname{sn}^{2}\left(\chi_{1}\right)$ and $\operatorname{sn}^{2}\left(\chi_{2}\right)$, up to $N_{\max }^{A^{\prime}}=N_{\max }^{B^{\prime}}$, respectively. The remaining task is to identify the harmonic linear superposition of the polynomials, making use of the linear independence and completeness of both bases in the second tool of the prelude. Some of our results on the actions of $\hat{L}_{x}$, $\hat{L}_{y}, \hat{L}_{z}$ on the non-normalized spheroconal harmonic polynomials $\ell A B n_{1} n_{2}$ for $\ell=0,1,2,3$ are illustrated and described next. The application of any of the operators $\hat{L}_{i}$ on the $\Lambda_{0}^{1} \Lambda_{0}^{1}=1$ leads to the same single eigenstate with its eigenvalue zero.

Table 4 exhibits the result of the corresponding applications on the three spheroconal harmonics with $\ell=1$. The contents in the table should not be surprising upon recognizing the species $x$, $y, z$ of the successive monomials, and their equivalence with the respective cartesian harmonics.

Table 5 for the $\ell=2$ polynomials contains familiar information for the unique monomial species $x y, x z$ and $y z$ transforming among themselves under the respective rotations, with coefficients one in the corresponding entries. The two $\Lambda_{0}^{1} \Lambda_{2}^{1}$ and $\Lambda_{2}^{1} \Lambda_{0}^{1}$ binomial companions get transformed into the monomials of the other species as illustrated by the top and bottom rows in the table, with coefficients involving the eigenvalues $h_{n_{1}}^{1}\left(k_{1}^{2}\right)$ and $h_{n_{2}}^{1}\left(k_{2}^{2}\right)$ of the matching binomials; the respective species of the monomials are complementary to that of the operators, as the reader can ascertain by moving along the top and bottom rows in the successive columns. In turn, the respective operators acting on the monomials lead to the linear superpositions of the two $\Lambda_{n_{1}}^{1} \Lambda_{n_{2}}^{1}$ binomials with more elaborate coefficients including the common one $C^{1}$

$$
\begin{aligned}
& C_{4,1}^{1}=\frac{-2 \bar{a}_{1}^{s}\left(h_{3}^{s}\left(k_{2}^{2}\right)\right)+\bar{a}_{1}^{d}\left(h_{0}^{d}\left(k_{1}^{2}\right)\right)}{\bar{a}_{1}^{d}\left(h_{2}^{d}\left(k_{1}^{2}\right)\right) \bar{a}_{1}^{s}\left(h_{3}^{s}\left(k_{2}^{2}\right)\right)-\bar{a}_{1}^{d}\left(h_{0}^{c}\left(k_{1}^{2}\right)\right) \bar{a}_{1}^{s}\left(h_{1}^{s}\left(k_{2}^{2}\right)\right)}, \quad C_{4,1}^{2}=1-C_{4,1}^{1} \\
& \left.C_{4,3}^{1}=C_{4,1}^{1} \text { (by changing } d \rightarrow s\right), \quad C_{4,3}^{2}=-\left(1+C_{4,3}^{1}\right), \\
& C_{4,2}^{1}=-C_{4,2}^{2}=\frac{\bar{a}_{1}^{c}\left(h_{2}^{c}\left(k_{2}^{2}\right)\right)+\bar{a}_{1}^{c}\left(h_{1}^{c}\left(k_{1}^{2}\right)\right)}{\bar{a}_{1}^{c}\left(h_{3}^{c}\left(k_{1}^{2}\right)\right) \bar{a}_{1}^{c}\left(h_{2}^{c}\left(k_{2}^{2}\right)\right)-\bar{a}_{1}^{c}\left(h_{1}^{c}\left(k_{1}^{2}\right)\right) \bar{a}_{1}^{c}\left(h_{0}^{c}\left(k_{2}^{2}\right)\right)} .
\end{aligned}
$$

The spheroconal harmonics with $\ell=3$ come in a single monomial of species $[d c s][s c d]=$ $[x y z]$ and three pairs of binomials of species $x, y$ and $z$. The monomial appears in the middle row of Table 6 , and its companions of the other species in the rows below and above. The monomial transformed into the $x, y, z$ species in the successive columns by the respective angular momentum components; the transformed states are linear combinations of the pair of 
Table 5. Eigenfunctions with $\ell=2$ and its resulting application of each cartesian angular momentum operator. The common factor in the table has the value $C^{1}=\left[h_{0}^{1}\left(k_{1}^{2}\right)-h_{2}^{1}\left(k_{1}^{2}\right)\right]^{-1}$.

\begin{tabular}{c|ccc} 
& $\hat{L}_{x}$ & $\hat{L}_{y}$ & $\hat{L}_{z}$ \\
\hline \hline$\Lambda_{0}^{1} \Lambda_{2}^{1}$ & $-h_{0}^{1}\left(k_{1}^{2}\right) \Lambda_{2}^{c s} \Lambda_{0}^{c d}$ & $\left(h_{0}^{1}\left(k_{1}^{2}\right)-h_{2}^{1}\left(k_{2}^{2}\right)\right) \Lambda_{1}^{d s} \Lambda_{1}^{s d}$ & $h_{2}^{1}\left(k_{2}^{2}\right) \Lambda_{1}^{d c} \Lambda_{1}^{s c}$ \\
$\Lambda_{1}^{d c} \Lambda_{1}^{s c}$ & $\Lambda_{1}^{d s} \Lambda_{1}^{s d}$ & $-\Lambda_{2}^{c s} \Lambda_{0}^{c d}$ & $C^{1}\left(\begin{array}{c}\left(2-h_{0}^{1}\left(k_{1}^{2}\right)\right) \Lambda_{2}^{1} \Lambda_{0}^{1}- \\
\left(2-h_{2}^{1}\left(k_{1}^{2}\right)\right) \Lambda_{0}^{1} \Lambda_{2}^{1}\end{array}\right)$ \\
$\Lambda_{1}^{d s} \Lambda_{1}^{s d}$ & $-\Lambda_{1}^{d c} \Lambda_{1}^{s c}$ & $2 C^{1}\left(\begin{array}{c}\Lambda_{2}^{1} \Lambda_{0}^{1} \\
-\Lambda_{0}^{1} \Lambda_{2}^{1}\end{array}\right)$ & $\Lambda_{2}^{c s} \Lambda_{0}^{c d}$ \\
$\Lambda_{2}^{c s} \Lambda_{0}^{c d}$ & $C^{1}\left(\begin{array}{c}\left(2-h_{2}^{1}\left(k_{2}^{2}\right)\right) \Lambda_{2}^{1} \Lambda_{0}^{1}- \\
\left(2-h_{0}^{1}\left(k_{2}^{2}\right)\right) \Lambda_{0}^{1} \Lambda_{2}^{1}\end{array}\right)$ & $\Lambda_{1}^{d c} \Lambda_{1}^{s c}$ & $-\Lambda_{1}^{d s} \Lambda_{1}^{s d}$ \\
$\Lambda_{2}^{1} \Lambda_{0}^{1}$ & $-h_{2}^{1}\left(k_{1}^{2}\right) \Lambda_{2}^{c s} \Lambda_{0}^{c d}$ & $\left(h_{2}^{1}\left(k_{1}^{2}\right)-h_{0}^{1}\left(k_{2}^{2}\right)\right) \Lambda_{1}^{d s} \Lambda_{1}^{s d}$ & $h_{0}^{1}\left(k_{2}^{2}\right) \Lambda_{1}^{d c} \Lambda_{1}^{s c}$
\end{tabular}

Table 6. Eigenfunctions with $\ell=3$ and its resulting application of each cartesian angular momentum operator. The element $C_{i, j}^{k}$ represents the $k$ coefficient of the linear combination at the $i$ row and $j$ column of the table. Some examples are represented in the equations (32)-(34).

\begin{tabular}{c|ccc} 
& $\hat{L}_{x}$ & $\hat{L}_{y}$ & $\hat{L}_{z}$ \\
\hline \hline$\Lambda_{0}^{d} \Lambda_{3}^{s}$ & $2 \bar{a}_{1}^{d}\left(h_{0}^{d}\left(k_{1}^{2}\right)\right) \Lambda_{2}^{d c s} \Lambda_{1}^{s c d}$ & $C_{1,2}^{1} \Lambda_{1}^{s} \Lambda_{2}^{d}+C_{1,2}^{2} \Lambda_{3}^{s} \Lambda_{0}^{d}$ & $C_{1,3}^{1} \Lambda_{1}^{c} \Lambda_{2}^{c}+C_{1,3}^{2} \Lambda_{3}^{c} \Lambda_{0}^{c}$ \\
$\Lambda_{1}^{c} \Lambda_{2}^{c}$ & $C_{2,1}^{1} \Lambda_{1}^{s} \Lambda_{2}^{d}+C_{2,1}^{2} \Lambda_{3}^{s} \Lambda_{0}^{d}$ & $\left(h_{1}^{c}\left(k_{1}^{2}\right)-h_{2}^{c}\left(k_{2}^{2}\right)\right) \Lambda_{2}^{d c s} \Lambda_{1}^{s c d}$ & $C_{2,3}^{1} \Lambda_{0}^{d} \Lambda_{3}^{s}+C_{2,3}^{2} \Lambda_{2}^{d} \Lambda_{1}^{s}$ \\
$\Lambda_{1}^{s} \Lambda_{2}^{d}$ & $C_{3,1}^{1} \Lambda_{1}^{c} \Lambda_{2}^{c}+C_{3,1}^{2} \Lambda_{3}^{c} \Lambda_{0}^{c}$ & $C_{3,2}^{1} \Lambda_{0}^{d} \Lambda_{3}^{s}+C_{3,2}^{2} \Lambda_{2}^{d} \Lambda_{1}^{s}$ & $-2 \bar{a}_{1}^{d}\left(h_{2}^{d}\left(k_{2}^{2}\right)\right) \Lambda_{2}^{d c s} \Lambda_{1}^{s c d}$ \\
$\Lambda_{2}^{d c s} \Lambda_{1}^{s c d}$ & $C_{4,1}^{1} \Lambda_{0}^{d} \Lambda_{3}^{s}+C_{4,1}^{2} \Lambda_{2}^{d} \Lambda_{1}^{s}$ & $C_{4,2}^{1} \Lambda_{1}^{c} \Lambda_{2}^{c}+C_{4,2}^{2} \Lambda_{3}^{c} \Lambda_{0}^{c}$ & $C_{4,3}^{1} \Lambda_{1}^{s} \Lambda_{2}^{d}+C_{4,3}^{2} \Lambda_{3}^{s} \Lambda_{0}^{d}$ \\
$\Lambda_{2}^{d} \Lambda_{1}^{s}$ & $2 \bar{a}_{1}^{d}\left(h_{2}^{d}\left(k_{1}^{2}\right)\right) \Lambda_{2}^{d c s} \Lambda_{1}^{s c d}$ & $C_{5,2}^{1} \Lambda_{1}^{s} \Lambda_{2}^{d}+C_{5,2}^{2} \Lambda_{3}^{s} \Lambda_{0}^{d}$ & $C_{5,3}^{1} \Lambda_{1}^{c} \Lambda_{2}^{c}+C_{5,3}^{2} \Lambda_{3}^{c} \Lambda_{0}^{c}$ \\
$\Lambda_{3}^{c} \Lambda_{0}^{c}$ & $C_{6,1}^{1} \Lambda_{1}^{s} \Lambda_{2}^{d}+C_{6,1}^{2} \Lambda_{3}^{s} \Lambda_{0}^{d}$ & $\left(h_{3}^{c}\left(k_{1}^{2}\right)-h_{0}^{c}\left(k_{2}^{2}\right)\right) \Lambda_{2}^{d c s} \Lambda_{1}^{s c d}$ & $C_{6,3}^{1} \Lambda_{0}^{d} \Lambda_{3}^{s}+C_{6,3}^{2} \Lambda_{2}^{d} \Lambda_{1}^{s}$ \\
$\Lambda_{3}^{s} \Lambda_{0}^{d}$ & $C_{7,1}^{1} \Lambda_{1}^{c} \Lambda_{2}^{c}+C_{7,1}^{2} \Lambda_{3}^{c} \Lambda_{0}^{c}$ & $C_{7,2}^{1} \Lambda_{0}^{d} \Lambda_{3}^{s}+C_{7,2}^{2} \Lambda_{2}^{d} \Lambda_{1}^{s}$ & $2 \bar{a}_{1}^{d}\left(h_{0}^{d}\left(k_{2}^{2}\right)\right) \Lambda_{2}^{d c s} \Lambda_{1}^{s c d}$
\end{tabular}

companion binomials with different number of nodes $n_{1}$ and $n_{2}$. The inverse transformations of the successive binomials leading back to the single monomial are identified in the row and column positions $(1,1),(2,2),(3,3),(5,1),(6,2)$ and $(7,3)$. The individual binomial spheroconal harmonics are transformed also into linear combinations of the pairs of binomials of species complementary to the component of the angular momentum, as an inspection of the remaining twelve positions in the table show. The coefficients involved become more numerous, taking into account the increasing number of the states and their combinations.

We complement this section with the following diagrams in Fig. 1 sketching the connections among the four species, for the two kinds of even and odd $\ell$ of spheroconal harmonics, provided by the angular momentum components. The interested readers may compare them with their counterparts in [24]. The coefficients in the entries of Tables 4-6 are the counterpart of the raising and lowering operator coefficients for the spherical harmonics.
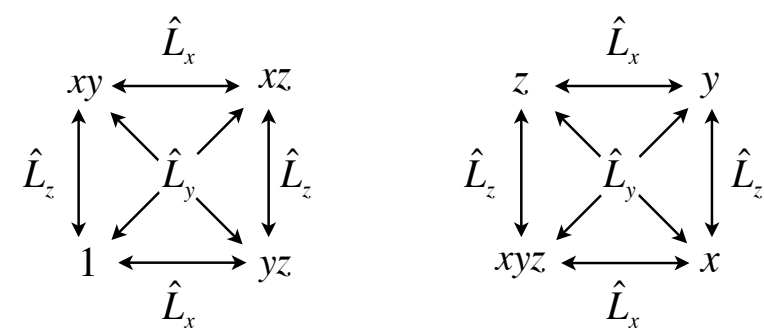

Figure 1. Action of the angular momentum operators on each of the spheroconal harmonics. 


\section{$5 \quad$ Linear momentum cartesian components raising and lowering the angular momentum by one unit between polynomials of opposite parities}

The cartesian components of the linear momentum operator in spheroconal coordinates are

$$
\begin{aligned}
& \hat{p}_{x}=\operatorname{dn}\left(\chi_{1}\right) \operatorname{sn}\left(\chi_{2}\right) \hat{p}_{r}-\frac{i \hbar r}{h_{\chi}^{2}}\left[-k_{1}^{2} \operatorname{sn}\left(\chi_{1}\right) \operatorname{cn}\left(\chi_{1}\right) \operatorname{sn}\left(\chi_{2}\right) \frac{\partial}{\partial \chi_{1}}+\operatorname{dn}\left(\chi_{1}\right) \operatorname{cn}\left(\chi_{2}\right) \operatorname{dn}\left(\chi_{2}\right) \frac{\partial}{\partial \chi_{2}}\right], \\
& \hat{p}_{y}=\operatorname{cn}\left(\chi_{1}\right) \operatorname{cn}\left(\chi_{2}\right) \hat{p}_{r}-\frac{i \hbar r}{h_{\chi}^{2}}\left[-\operatorname{sn}\left(\chi_{1}\right) \operatorname{dn}\left(\chi_{1}\right) \operatorname{cn}\left(\chi_{2}\right) \frac{\partial}{\partial \chi_{1}}-\operatorname{cn}\left(\chi_{1}\right) \operatorname{sn}\left(\chi_{2}\right) \operatorname{dn}\left(\chi_{2}\right) \frac{\partial}{\partial \chi_{2}}\right], \\
& \hat{p}_{z}=\operatorname{sn}\left(\chi_{1}\right) \operatorname{dn}\left(\chi_{2}\right) \hat{p}_{r}-\frac{i \hbar r}{h_{\chi}^{2}}\left[\operatorname{cn}\left(\chi_{1}\right) \operatorname{dn}\left(\chi_{1}\right) \operatorname{dn}\left(\chi_{2}\right) \frac{\partial}{\partial \chi_{1}}-k_{2}^{2} \operatorname{sn}\left(\chi_{1}\right) \operatorname{sn}\left(\chi_{2}\right) \operatorname{cn}\left(\chi_{2}\right) \frac{\partial}{\partial \chi_{2}}\right]
\end{aligned}
$$

Notice that the angular factors of $\hat{p}_{r}$ are simply the projections of the unit radial vector along the respective axes, equation (4). The angular factor of the derivatives inside the brackets in equations (29)-(31) and (35)-(37) for the successive components are the same but in exchanged positions, and different signs in some cases. The reason for this is found in the decomposition of the $\vec{p}$ vector into its radial and transverse components

$$
\hat{\vec{p}}=\hat{r} \hat{p}_{r}-\vec{r} \times \frac{\hat{\vec{L}}}{r} .
$$

already used in [18]. Correspondingly, also notice the presence of the factor $r / h_{\chi}^{2}$ in equations (35)-(37). The actions of these on a spheroconal harmonic functions $\ell A B n_{1} n_{2}$ lead to functions of the other kind $\ell^{\prime} A^{\prime} B^{\prime} n_{1}^{\prime} n_{2}^{\prime}$ with $\ell^{\prime}=\ell \pm 1$, species $A^{\prime} B^{\prime}$ with the other parity. Consequently, the method and some of the steps and results of the previous section are useful or have their counterparts in the application of the operators of equations (35)-(37) on the spheroconal harmonic polynomials $\ell A B n_{1} n_{2}$.

At the level of each individual spheroconal coordinate, the multiplications by $d, c$, and $s$ of the first terms in the operators in equations (35)-(37) with the eight species of singularity removing factors $A(\chi)$ lead to the following result:

\begin{tabular}{l|llllllll} 
& 1 & $s$ & $c$ & $d$ & $c d$ & $s d$ & $s c$ & $s c d$ \\
\hline \hline$d$ & $d$ & $d s$ & $d c$ & $d^{2}$ & $c d^{2}$ & $s d^{2}$ & $s c d$ & $s c d^{2}$ \\
$c$ & $c$ & $c s$ & $c^{2}$ & $c d$ & $c^{2} d$ & $s c d$ & $s c^{2}$ & $s c^{2} d$ \\
$s$ & $s$ & $s^{2}$ & $s c$ & $s d$ & $s c d$ & $s^{2} d$ & $s^{2} c$ & $s^{2} c d$
\end{tabular}

with the common result that every original $A(\chi)$ is changed in kind and species. The effects are qualitatively similar and complementary to those of the first derivative in the table of the prelude at the end of Section 3. In fact, the union of both tables provides the sets of four species associated with the other kind of Lamé functions.

What has been said for $A\left(\chi_{1}\right)$ also holds for the companion $B\left(\chi_{2}\right)$, and the resulting $A^{\prime}\left(\chi_{1}\right)$ and $B^{\prime}\left(\chi_{2}\right)$ can be properly matched. At the level of multiplication in the first terms of equations (35)-(37), their respective $x, y$ and $z$ character has already been recognized. As a consequence of the exchange of angular factors in the derivative terms in equations (29)-(31) and (35)-(37), the kinds and species maintained in the previous section are changed in the present one. The net result is that the angular derivative terms in equations (35)-(37) acting on the individual Lamé functions $\ell A n_{1}$ and $\ell B n_{2}$ transform them into the same kinds and species $A^{\prime}\left(\chi_{1}\right), B^{\prime}\left(\chi_{2}\right)$ obtained by multiplication in the respective first terms. 
We proceed to analyze the effects of the operators of equations (35)-(37) on the successive Lamé spheroconal functions. For $\ell=0, \Lambda_{0}^{1}\left(\chi_{1}\right) \Lambda_{0}^{1}\left(\chi_{2}\right)=1$ is annihilated by the derivative terms, and transformed into

$$
\begin{aligned}
& \operatorname{dn}\left(\chi_{1}\right) \operatorname{sn}\left(\chi_{2}\right)=\Lambda_{0}^{d}\left(\chi_{1}\right) \Lambda_{1}^{s}\left(\chi_{2}\right)=\frac{x}{r}, \quad \operatorname{cn}\left(\chi_{1}\right) \operatorname{cn}\left(\chi_{2}\right)=\Lambda_{1}^{c}\left(\chi_{1}\right) \Lambda_{0}^{c}\left(\chi_{2}\right)=\frac{y}{r}, \\
& \operatorname{sn}\left(\chi_{1}\right) \operatorname{dn}\left(\chi_{2}\right)=\Lambda_{1}^{s}\left(\chi_{1}\right) \Lambda_{0}^{d}\left(\chi_{2}\right)=\frac{z}{r},
\end{aligned}
$$

respectively, by the multiplication operations. The identifications with the cartesian harmonics is also made. Their angular momentum of $\ell=1$ and negative parity are also recognized in contrast with the $\ell=0$ and positive parity of the original state.

Next, we illustrate the net result of the applying the derivative terms on the $\ell=1$ states, reducing them by factoring the square of the scale factor, obtaining the following table of monomials and binomials:

Table 7. Actions of angular derivative terms in $\hat{p}_{x}, \hat{p}_{y}, \hat{p}_{z}$ on $\ell=1$ spheroconal harmonics.

\begin{tabular}{c|ccc} 
& $\hat{p}_{x}$ & $\hat{p}_{y}$ & $\hat{p}_{z}$ \\
\hline \hline$\Lambda_{0}^{1 d} \Lambda_{1}^{1 s}$ & $1-\operatorname{dn}^{2}\left(\chi_{1}\right) \operatorname{sn}^{2}\left(\chi_{2}\right)$ & $\Lambda_{1}^{2 d c} \Lambda_{1}^{2 s c}$ & $\Lambda_{1}^{2 d s} \Lambda_{1}^{2 s d}$ \\
$\Lambda_{1}^{1 c} \Lambda_{0}^{1 c}$ & $\Lambda_{1}^{2 d c} \Lambda_{1}^{2 s c}$ & $1-\operatorname{cn}^{2}\left(\chi_{1}\right) \mathrm{cn}^{2}\left(\chi_{2}\right)$ & $\Lambda_{2}^{2 c s} \Lambda_{0}^{2 c d}$ \\
$\Lambda_{1}^{1 s} \Lambda_{0}^{1 d}$ & $\Lambda_{1}^{2 d s} \Lambda_{1}^{2 s d}$ & $\Lambda_{2}^{2 c s} \Lambda_{0}^{2 c d}$ & $1-\operatorname{sn}^{2}\left(\chi_{1}\right) \operatorname{dn}^{2}\left(\chi_{2}\right)$
\end{tabular}

The off-diagonal entries are identified as $\Lambda_{1}^{2 d c}\left(\chi_{1}\right) \Lambda_{1}^{2 s c}\left(\chi_{2}\right)=x y, \Lambda_{1}^{2 d s}\left(\chi_{1}\right) \Lambda_{1}^{2 s d}\left(\chi_{2}\right)=x z$ and $\Lambda_{2}^{2 c s}\left(\chi_{1}\right) \Lambda_{0}^{2 c d}\left(\chi_{2}\right)=y z$ obtained via two different operators acting on two different initial states. They could also be obtained via the multiplication with the factors in the first terms of equations (35)-(36). The identification of their angular momentum $\ell=2$, and positive parity and nodal quantum numbers can be made from Table 3.

The diagonal entries deserve additional analysis before the lowering effect of the operators is identified, as well as the raising effects leading to the remaining two $\ell=2$ spheroconal harmonics. The three entries have been obtained via three different operators acting on three different initial functions. Of course, the "ones" correspond to the monopolar harmonic to which we go back by the lowering effect on the dipole harmonics. On the other hand, the other terms are identified as the squares of the projections of the radial unit vector along the coordinate axes. Their sum is "one" coinciding again with the lowered $\ell=0$ state. Of the three terms, only two are linearly independent, since their sum is fixed. This suggests that the three terms can be combined in two linearly independent combinations to be identified with the missing $\Lambda_{0}^{21}\left(\chi_{1}\right) \Lambda_{2}^{21}\left(\chi_{2}\right)$ and $\Lambda_{2}^{21}\left(\chi_{1}\right) \Lambda_{0}^{21}\left(\chi_{2}\right), \ell=2$ spheroconal harmonics.

The analysis can be made more transparent by recognizing that $x^{2}, y^{2}$ and $z^{2}$ are equivalent to them upon multiplication by $r^{2}$. Let us consider the linear combination

$$
\Phi(x, y, z)=\alpha x^{2}+\beta y^{2}+\gamma z^{2} .
$$

It is advantageous to choose it as harmonic

$$
\nabla^{2} \Phi=2 \alpha+2 \beta+2 \gamma=0
$$

because in such a case we are sure that their connection with the spheroconal harmonics is linear. In fact, from equation (40) we get

$$
\beta=-\alpha-\gamma,
$$

and rewrite equation (39) in the separable form in spheroconal coordinates

$$
\Phi\left(r, \chi_{1}, \chi_{2}\right)=r^{2}\left[\alpha \operatorname{dn}^{2}\left(\chi_{1}\right) \mathrm{cn}^{2}\left(\chi_{2}\right)-(\alpha+\gamma) \mathrm{cn}^{2}\left(\chi_{1}\right) \mathrm{cn}^{2}\left(\chi_{2}\right)+\gamma \operatorname{sn}^{2}\left(\chi_{1}\right) \operatorname{dn}^{2}\left(\chi_{2}\right)\right] .
$$


The question to be answered is which values of the coefficients $\alpha$ and $\gamma$ combining the diagonal angular entries lead to the $\ell=2$ spheroconal harmonics of species 11 .

The answers are obtained by rewriting equation (41) as polynomials in even-powers of $\operatorname{sn}\left(\chi_{1}\right)$ and $\operatorname{sn}\left(\chi_{2}\right)$ and comparing them with the target polynomials from Table 2. Their explicit forms are

$$
\alpha_{02}=-\frac{1}{2}+\frac{1}{4} h_{0}^{1}\left(k_{1}^{2}\right)-\frac{1}{4} h_{2}^{1}\left(k_{2}^{2}\right), \quad \gamma_{02}=-\frac{1}{2}-\frac{1}{4} h_{0}^{1}\left(k_{1}^{2}\right)+\frac{1}{4} h_{2}^{1}\left(k_{2}^{2}\right),
$$

and

$$
\alpha_{20}=-\frac{1}{2}+\frac{1}{4} h_{2}^{1}\left(k_{1}^{2}\right)-\frac{1}{4} h_{0}^{1}\left(k_{2}^{2}\right), \quad \gamma_{20}=-\frac{1}{2}-\frac{1}{4} h_{2}^{1}\left(k_{1}^{2}\right)+\frac{1}{4} h_{0}^{1}\left(k_{2}^{2}\right),
$$

defining the proportions of the presence of the respective spheroconal harmonics in the diagonal entries in the Table 7 . We remind the reader that the analysis has been made for non-normalized polynomials. In any case, the coefficients above are the counterpart of the familiar spherical Clebsch-Gordan coefficients. Work on the raising and lowering from $\ell=2,3, \ldots$ is in process.

\section{Discussion}

Some of our works on the rotations of asymmetric molecules and the Lamé spheroconal harmonics have taken advantage of their connections with their spherical and cartesian counterparts [9, 18], and others have been developed strictly within the spheroconal formalism [10, 17, 19, 18]. The analysis and identification of the three sets of ladder operators in this article has been purposefully implemented in the same formalism, avoiding the spherical and cartesian crutches.

The connections among Lamé spheroconal polynomials of a given kind and species, and different numbers of nodes have been explicitly recognized in Section 3. They share the same form and degree, differing in their number of nodes $n_{i}$; in contrast with the classical polynomials having a number of nodes equal to their degree. For the spheroconal harmonics constructed by the product of Lamé polynomials with matching number of nodes and eigenvalues, equations (22) and (23), their complete set is connected by their complementary raising of $n_{1}$ and lowering of $n_{2}$. The inversion of equation (25) provides the basis of the powers of $\operatorname{sn}^{2}(\chi)$ as a linear combination of the set of corresponding Lamé polynomials, as the counterpart of the known relationships in the classical polynomials.

Our other contribution in this volume of SIGMA [11] contains another example of polynomial eigenfunctions, for the Hydrogen atom confined by a dihedral angle in prolate spheroidal coordinates, evaluated by diagonalization of tridiagonal finite size matrices, and their connections by complementary changes in the numbers of prolate spheroidal and hyperboloidal nodes.

The shifting actions of the $\hat{L}_{x}, \hat{L}_{y}, \hat{L}_{z}$ operators among the spheroconal harmonics of the four species for a chosen value $\ell$, as illustrated in Tables 5 and 6 , allow the generation of the corresponding coefficients in the purely spheroconal formalism. They are the counterpart of the familiar raising and lowering coefficients $C_{ \pm}(\ell, m)$ for the spherical harmonics, and even closer for the spherical harmonics with definite parities. The relationships so far are for the non-normalized coefficients, but work is under way to identify and incorporate the normalization coefficients.

The $\ell$ raising and lowering actions of $\hat{p}_{x}, \hat{p}_{y}$ and $\hat{p}_{z}$ among spheroconal harmonics of neighboring kinds and species has been illustrated for the lower states, but the systematics had already been recognized in [18]. While writing the latter, several works on related topics $[12,30,31]$ allowed us to recognize the complete radial and angular raising and lowering actions for the free particle in spherical coordinates. Now we can also extend it to spheroconal coordinates.

Piña identified four step $\Delta \ell=4$ ladder operators for spheroconal harmonics of the same species for the most asymmetric molecules [26]. We are investigating their possible connection 
with the application of the $\hat{p}_{i}$ operators four times in succession leading to the same states. In such a case the results are valid for any asymmetry distribution.

The familiarity with cartesian, spherical and spheroconal harmonics is decreasing when written in this order. The works $[9,24]$ used the spherical basis as the tool to evaluate the spheroconal harmonics. In the process the interbasis expansion coefficients are generated for the original problem, and automatically also for the inverse problem. The specific example at the end of Section 5 shows the spheroconal quadrupole composition of the cartesian one in equation (39).

As a complement to the comment in the third paragraph of this section, we include the following related references with their respective comments. The contents in [2] for the free Hydrogen atom in configuration space overlap with ours in [11], with emphasis on the rarely treated spheroidal sets, and in the present contribution, on the properties of spheroelliptic orbitals, which have been so far practically ignored. The related work [3] also deals with the free Hydrogen atom in momentum space focusing on the hyperspherical harmonic elliptic Sturmian basis set in $S^{3}$. The earlier work [5] deals with the free quantum motion on the three-dimensional sphere $S^{3}$ ellipso-cyllindrical coordinates, distinguishing between prolate and oblate elliptic coordinates. On the other hand the more recent work [4] deals with the use of the hyperspherical elliptic coordinates and harmonic basis sets in the fifth dimensional sphere $S^{5}$ for the study of the three-body problem in quantum mechanics. Its classical astronomical counterpart [28] uses the relative radial coordinate of the first two particles and the radial coordinate of the third particle relative to the center of mass of the first two, instead of the hyper-radius and one of the five angular coordinates in $S^{5}$. It is also pertinent to mention Sasaki's contribution in the Symposium, "Exactly solvable quantum mechanics and infinite families of multi-indexed orthogonal polynomials", and to cite [22, 23], involving Hamiltonians with tridiagonal matrix representations. The raising and lowering operators in our Section 3 have their counterparts for the respective eigenfunctions in the references of this paragraph.

\section{Acknowledgements}

The authors express their appreciation to the organizers of the Symposium and editors of this Volume of SIGMA on "Superintegrability, Exact Solvability, and Special Functions" for their invitations to participate in both. The authors acknowledge the financial support for this work by Consejo Nacional de Ciencia y Tecnología, SNI-1796.

\section{References}

[1] Abramowitz M., Stegun I.A., Handbook of mathematical functions, Dover, New York, 1965.

[2] Aquilanti V., Caligiana A., Cavalli S., Hydrogenic elliptic orbitals, Coulomb Sturmian sets, and recoupling coefficients among alternative bases, Int. J. Quantum Chem. 92 (2003), 99-117.

[3] Aquilanti V., Caligiana A., Cavalli S., Coletti C., Hydrogenic orbitals in momentum space and hyperspherical harmonics: elliptic Sturmian basis sets, Int. J. Quantum Chem. 92 (2003), 212-228.

[4] Aquilanti V., Tonzani S., Three-body problem in quantum mechanics: hyperspherical elliptic coordinates and harmonic basis sets, J. Chem. Phys. 120 (2004), 4066-4073.

[5] Grosche C., Karayan K.H., Pogosyan G.S., Sissakian A.N., Quantum motion on the three-dimensional sphere: the ellipso-cylindrical bases, J. Phys. A: Math. Gen. 30 (1997), 1629-1657.

[6] Kramers H.A., Ittmann G.P., Zur Quantelung des asymmetrischen Kreisels, Z. Phys. 53 (1929), 553-565.

[7] Kronig R. de L., Rabi I.I., The symmetrical top in the undulatory mechanics, Phys. Rev. 29 (1927), $262-269$.

[8] Kroto H.W., Molecular rotation spectra, John Wiley \& Sons, London, 1975.

[9] Ley-Koo E., Méndez-Fragoso R., Properties of the spectra of asymmetric molecules: matrix evaluation in bases of spherical harmonics and common generating function, Rev. Mexicana Fís. 54 (2008), 69-77. 
[10] Ley-Koo E., Méndez-Fragoso R., Rotational states of asymmetric molecules revisited: matrix evaluation and generating function of Lamé functions, Rev. Mexicana Fís. 54 (2008), 162-172.

[11] Ley-Koo E., Sun G.H., Ladder operators for quantum systems confined by dihedral angles, SIGMA 8 (2012), 060, 15 pages, arXiv:1209.2497.

[12] Liu Q.H., Xun D.M., Shan L., Raising and lowering operators for orbital angular momentum quantum numbers, Internat. J. Theoret. Phys. 49 (2010), 2164-2171.

[13] Lukach I., A complete set of quantum-mechanical observables on a two-dimensional sphere, Theoret. and Math. Phys. 14 (1973), 271-281.

[14] Lukach I., Smorodinskiŭ Ya.A., Separation of variables in a spheroconical coordinate system and the Schrödinger equation for a case of noncentral forces, Theoret. and Math. Phys. 14 (1973), 125-131.

[15] Lukach I., Smorodinskiu Ya.A., The wave functions of an asymmetrical top, Soviet Phys. JETP 30 (1970), 728-730.

[16] Lütgemeier F., Zur Quantentheorie des drei- und mehratomigen Moleküls, Z. Phys. 38 (1926), $251-263$.

[17] Méndez-Fragoso R., Ley-Koo E., Lamé spheroconal harmonics in atoms and molecules, Int. J. Quantum Chem. 110 (2010), 2765-2774.

[18] Méndez-Fragoso R., Ley-Koo E., Rotations of asymmetric molecules and the hydrogen atom in free and confined congurations, Adv. Quantum Chem. 62 (2011), 137-213.

[19] Méndez-Fragoso R., Ley-Koo E., The hydrogen atom in a semi-infinite space with an elliptical cone boundary, Int. J. Quantum Chem. 111 (2011), 2882-2897.

[20] Morse P.M., Feshbach H., Methods of theoretical physics, Vols. 1, 2, McGraw-Hill Book Co. Inc., New York, 1953.

[21] Niven W.D., On ellipsoidal harmonics, Philos. Trans. R. Soc. Lond. Ser. A 182 (1891), 231-278.

[22] Odake S., Sasaki R., Discrete quantum mechanics, J. Phys. A: Math. Theor. 44 (2011), 353001, 47 pages, arXiv:1104.0473.

[23] Odake S., Sasaki R., Orthogonal polynomials from Hermitian matrices, J. Math. Phys. 49 (2008), 053503, 43 pages, arXiv:0712.4106.

[24] Patera J., Winternitz P., A new basis for the representations of the rotation group. Lamé and Heun polynomials, J. Math. Phys. 14 (1973), 1130-1139.

[25] Patera J., Winternitz P., On bases for irreducible representations of $O(3)$ suitable for systems with an arbitrary finite symmetry group, J. Chem. Phys. 65 (1976), 2725-2731.

[26] Piña E., Algunas propiedades de los operadores de escalera, Rev. Mexicana Fís. 41 (1995), 913-924.

[27] Piña E., Some properties of the spectra of asymmetric molecules, J. Mol. Structure: THEOCHEM 493 (1999), 159-170.

[28] Piña E., Jiménez-Lara L., Properties of new coordinates for the general three-body problem, Celestial Mech. Dynam. Astronom. 82 (2002), 1-18.

[29] Reiche F., Rademacher H., Die Quantelung des symmetrischen Kreisels nach Schrödingers Undulationsmechanik, Z. Phys. 39 (1926), 444-464.

[30] Sun G.H., Dong S.H., New type shift operators for circular well potential in two dimensions, Phys. Lett. A 374 (2010), 4112-4114.

[31] Sun G.H., Dong S.H., New type shift operators for three-dimensional infinite well potential, Modern Phys. Lett. A 26 (2011), 351-358.

[32] Valdéz M.T., Piña E., The rotational spectra of the most asymmetric molecules, Rev. Mexicana Fís. 52 (2006), 220-229.

[33] Volkmer H., Lamé functions, in NIST Handbook of Mathematical Functions, Editors F.W.J. Olver, D.W. Lozier, R.F. Boisvert, C.W. Clark, U.S. Dept. Commerce, Washington, DC, 2010, 683-695.

[34] Whittaker E.T., Watson G.N., A course of modern analysis, Cambridge Mathematical Library, Cambridge University Press, Cambridge, 1996.

[35] Witmer E.E., The rotational energy of the polyatomic molecule as an explicit function of the quantum numbers, Proc. Natl. Acad. Sci. USA 12 (1926), 602-608. 